九州大学学術情報リポジトリ

Kyushu University Institutional Repository

\title{
On Behaviors of Cellular Automata with Rule 156
}

Inokuchi, Shuichi

Department of Informatics, Kyushu University

http://hdl. handle. net/2324/3014

出版情報 : DOI Technical Report. 146，1998-03-11. Department of Informatics, Kyushu University バージョン：

権利関係 : 
DOI-TR-146

\title{
DOI Technical Report
}

\section{On Behaviors of Cellular Automata with Rule 156}

\author{
by
}

ShUICHI INOKUCHI

\author{
March 11, 1998
}

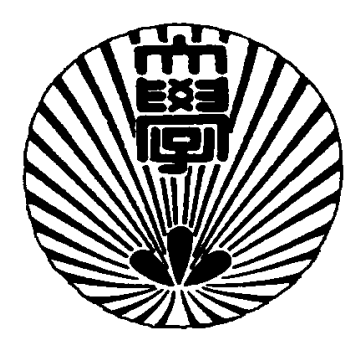

Department of Informatics

Kyushu University

Fukuoka 812-81, Japan 



\title{
On Behaviors of Cellular Automata with Rule 156
}

\author{
Shuichi INOKUCHI *
}

\author{
March 11, 1998
}

\begin{abstract}
This paper deals with one-dimensional finite cellular automata with a triplet local transition rule 156 and fixed boundary conditions. The author observed behaviors of CA-156 $6_{0-0}(m)$ by computer, and found formulae of number of limit cycles and transient length from observation. And the author got proof of them.
\end{abstract}

\section{Introduction}

J. von Neumann introduced cellular automata capable of self-reproduction and universal computation. While cellular automata have simple structure, their global behaviors caused by interactions between cells are very complicated. Their global behaviors are similar to behaviors of complex systems as fractals, chaotic phenomenon and so on. S. Wolfram and other researchers rerealized important roles of cellular automata as theoretical models of complex systems. Cellular automata have wide applications in biology, physics, computer science, mathematics and so on.

Recently various cellular automata are investigated and applied by many researchers. S. Wolfram classified 1-dimensional cellular automata into 4 classes [1]. The author et al. simulated 1-dimensional cellular automata by computer and analyzed the result of the simulations [8]. Y. Kawahara et al. studied 2-dimensional cellular automata with linear rule 90 [2]. H. Lee and Y. Kawahara investigated 1-dimensional cellular automata with linear rule 60 [3]. M. Kawanaka et al. designed parallel processor model by a 2-dimensional cellular automata [5]. Many linear cellular automata are investigated and applied by many researchers. But many non-linear cellular automata have not investigated yet. Some non-linear cellular automata were investigated. T. Sato investigated behaviors of non-linear cellular automata with rule 27 [7]. And $\mathrm{H}$. Lee et al. investigated transition diagrams of 1-dimensional cellular automata and proved that transition diagrams of a cellular automaton can be represented by the cartesian product of transition diagrams of smaller cellular automat [6]. R. Shingai

* Department of Informatics, Kyushu University 33, Fukuoka 812-8581, Japan E-mail: inokuchi@i.kyushu-u.ac.jp 
showed that the period length of limit cycles of cellular automata with a threshold transition rule is 4 or less [4].

In this paper the author investigate 1-dimensional cellular automata with nonlinear transition rule 156 . And the author prove the formulas of the number of limit cycles found by analysing the result of computer simulations in the paper written by the author, T. Sato et al. [8].

\section{1-D Finite Cellular Automata}

A configuration is a set of cells arrayed and having a state. The set of all configurations is called a configuration set. A cellular automaton is a dynamical system $(X, f)$ of a configuration set $X$ and a transition function $f$ on $X$. A cellular automaton is 1-dimensional if cells of its configurations are arrayed linearly.

In this section, we define 1-dimensional finite cellular automata with a triplet local transition rule 156 and fixed boundary conditions and necessary notations for after discussion.

Definition 1 Let $\{1,2, \cdots, m\}$ be a set of cells. A configuration is a vector $x=$ $\left(x_{1}, x_{2}, \cdots, x_{m}\right)$ where $x_{i} \in\{0,1\}$. And we call the set of all configurations a configuration set.

Usually a configuration $\left(x_{1}, x_{2}, \cdots, x_{m}\right)$ is denoted by the sequence $x_{1} x_{2} \cdots x_{m}$ for short.

Definition 2 The triplet local transition rule $f$ is a function $\{0,1\}^{3} \longrightarrow\{0,1\}$, and $f$ is illustrated as follows;

$$
\left|\begin{array}{cccccccc}
111 & 110 & 101 & 100 & 011 & 010 & 001 & 000 \\
r_{7} & r_{6} & r_{5} & r_{4} & r_{3} & r_{2} & r_{1} & r_{0}
\end{array}\right|
$$

where $r_{i}=f(x y z)$ and $i=4 x+2 y+z$. And the rule number $R$ of $f$ is defined as follows;

$$
R=2^{7} r_{7}+2^{6} r_{6}+\cdots+2^{0} r_{0}
$$

For instance,

$$
156=2^{7} \times 1+2^{4} \times 1+2^{3} \times 1+2^{2} \times 1,
$$

so the triplet local transition rule $f$ of rule number 156 is given as follows;

$$
\text { Rule } 156\left|\begin{array}{cccccccc}
111 & 110 & 101 & 100 & 011 & 010 & 001 & 000 \\
1 & 0 & 0 & 1 & 1 & 1 & 0 & 0
\end{array}\right|
$$

Definition 3 The global transition function $\delta:\{0,1\}^{m} \longrightarrow\{0,1\}^{m}$ is defined as follows;

$$
\delta\left(x_{1}, x_{2}, \cdots, x_{m}\right)=\left(f\left(\alpha, x_{1}, x_{2}\right), f\left(x_{1}, x_{2}, x_{3}\right), \cdots, f\left(x_{m-1}, x_{m}, \beta\right)\right)
$$

where $f$ is a triplet local transition rule and $\alpha, \beta \in\{0,1\}$. 
We call a pair $(\alpha, \beta)$ a boundary condition. We say that the boundary condition is cyclic if and only if $\alpha=x_{m}$ and $\beta=x_{1}$, and the boundary condition is fixed if and only if $\alpha$ and $\beta$ are fixed. In particular,when $\alpha=a$ and $\beta=b$, we say that the boundary condition is $a-b$.

A 1-dimensional cellular automaton with $\mathrm{m}$ cells, rule $R$ and boundary condition $\alpha-\beta$ is denoted by $C A-R_{\alpha-\beta}(m)$.

Definition 4 Let $x$ be a configuration of $C A-R_{\alpha-\beta}(m)$. The configuration $x$ is on a limit cycle of period length $T$ if and only if there exists a positive integer s such that $\delta^{s}(x)=x$, and $T=\min \left\{s \geq 1 \mid \delta^{s}(x)=x\right\}$. And the configurations $x(1), x(2), \cdots, x(T-$ 1) form a limit cycle of period length $T$ if and only if $x(i+1)=\delta(x(i))$ and $x(T)=$ $x(1)$, and $x(i)$ forms a limit cycle of period length $T$ where $1 \leq i \leq T-1$.

A limit cycle of period length $T$ is called $T$-cycle, in particular, a limit cycle of period length 1 is called a fixed point. And $\gamma_{T}(m)$ denote a number of a limit cycle of period length $T$ of $C A-R_{\alpha-\beta}(m)$.

Definition 5 The height $h(x)$ of a configuration $x$ is defined as follows;

$$
h(x)=\min \left\{s \geq 0 \mid \delta^{s}(x) \text { is on a limit cycle }\right\} .
$$

Then the transient length $H(m)$ of $C A-R_{\alpha-\beta}(m)$ is defined as follows;

$$
H(m)=\max \left\{h(x) \mid x \text { is a configuration of } C A-R_{\alpha-\beta}(m)\right\}
$$

Definition 6 The reverse transition rule $\bar{f}$ of a local transition rule $f$ and the symmetric transition rule $f^{t}$ of $f$ is defined as follows;

$$
\begin{gathered}
\bar{f}(x, y, z)=1-f(1-x, 1-y, 1-z) \\
f^{t}(x, y, z)=f(z, y, x)
\end{gathered}
$$

Let $\bar{R}$ and $R^{t}$ be rule number of $\bar{f}$ and $f^{t}$ respectively, then we can identify $C A$ $\bar{R}_{\bar{\alpha}-\bar{\beta}}(m)$ and $C A-R_{\beta-\alpha}^{t}(m)$ with $C A-R_{\alpha-\beta}(m)$. So $C A-R_{\alpha-\beta}(m), C A-\bar{R}_{\bar{\alpha}-\bar{\beta}}(m), C A-$ $R_{\beta-\alpha}^{t}(m)$ and $C A-\bar{R}_{\bar{\beta}-\bar{\alpha}}(m)$ can be identified with each other.

The symmetric rule and the reverse rule of rule 156 are rule 198 illustrated as follows;

$$
\text { Rule } 198\left|\begin{array}{cccccccc}
111 & 110 & 101 & 100 & 011 & 010 & 001 & 000 \\
1 & 1 & 0 & 0 & 0 & 1 & 1 & 0
\end{array}\right|
$$

We use the following notations:

Let $A$ be a subsequence of a configuration of cellular automata.

- $A^{k}$ : sequence composed of $k A^{\prime}$ 's

- * : an irrelevant bit 


\section{Computer Simulations}

In this section we see the result of computer simulations of CA-156 $6_{\alpha-\beta}(m)$. The author simulated CA-156 $6_{\alpha-\beta}(m)$ by computer and got the following tables.

\begin{tabular}{|c||c|c|c|c|c|c|c|c|c|c|c|c|c|c|c|}
\hline $\mathrm{m}$ & 1 & 2 & 3 & 4 & 5 & 6 & 7 & 8 & 9 & 10 & 11 & 12 & 13 & 14 & 15 \\
\hline \hline 1-cycle & 2 & 2 & 3 & 3 & 4 & 4 & 5 & 5 & 6 & 6 & 7 & 7 & 8 & 8 & 9 \\
\hline 2-cycle & 0 & 1 & 1 & 3 & 5 & 8 & 14 & 22 & 34 & 55 & 83 & 129 & 199 & 302 & 464 \\
\hline $\mathrm{H}(\mathrm{m})$ & 0 & 0 & 2 & 2 & 4 & 4 & 6 & 6 & 8 & 8 & 10 & 10 & 12 & 12 & 14 \\
\hline
\end{tabular}

Table1:CA-156 $6_{0-0}(m)$ and CA-156 $6_{1-1}(m)$

\begin{tabular}{|c||c|c|c|c|c|c|c|c|c|c|c|c|c|c|c|}
\hline $\mathrm{m}$ & 1 & 2 & 3 & 4 & 5 & 6 & 7 & 8 & 9 & 10 & 11 & 12 & 13 & 14 & 15 \\
\hline \hline 1-cycle & 2 & 4 & 6 & 9 & 12 & 16 & 20 & 25 & 30 & 36 & 42 & 49 & 56 & 64 & 72 \\
\hline 2-cycle & 0 & 0 & 1 & 2 & 5 & 10 & 18 & 32 & 54 & 88 & 143 & 226 & 355 & 554 & 856 \\
\hline $\mathrm{H}(\mathrm{m})$ & 0 & 0 & 0 & 2 & 2 & 4 & 4 & 6 & 6 & 8 & 8 & 10 & 10 & 12 & 12 \\
\hline
\end{tabular}

Table1:CA-156-1 $(m)$

\begin{tabular}{|c||c|c|c|c|c|c|c|c|c|c|c|c|c|c|c|}
\hline $\mathrm{m}$ & 1 & 2 & 3 & 4 & 5 & 6 & 7 & 8 & 9 & 10 & 11 & 12 & 13 & 14 & 15 \\
\hline \hline 1-cycle & 0 & 1 & 0 & 1 & 0 & 1 & 0 & 1 & 0 & 1 & 0 & 1 & 0 & 1 & 0 \\
\hline 2-cycle & 1 & 0 & 2 & 2 & 3 & 6 & 8 & 12 & 21 & 28 & 46 & 70 & 103 & 162 & 244 \\
\hline $\mathrm{H}(\mathrm{m})$ & 0 & 2 & 2 & 4 & 4 & 6 & 6 & 8 & 8 & 10 & 10 & 12 & 12 & 14 & 14 \\
\hline
\end{tabular}

Table1:CA-156 $6_{1-0}(m)$

The numbers of limit cycles look irregular. But by analyzing the above tables by computer the following formulas are found. For CA-156 $6_{0-0}(m)$ and CA-156 $1-1(\mathrm{~m})$

$$
\begin{gathered}
\gamma_{1}(m)=\left[\frac{m-1}{2}\right]+2, \\
\gamma_{2}(m)=\gamma_{2}(m-1)+\gamma_{2}(m-2)+\gamma_{2}(m-3)-2 \gamma_{2}(m-4)+\left\{\begin{array}{ll}
0 & \text { if } m \text { is even } \\
1 & \text { otherwise }
\end{array},\right. \\
H(m)=2\left[\frac{m-1}{2}\right] .
\end{gathered}
$$

where [-] denotes the Gauss symbol.

For CA- $156_{0-1}(m)$

$$
\begin{gathered}
\gamma_{1}(m)=\gamma_{1}(m-1)+\gamma_{1}(m-2)-\gamma_{1}(m-3)+1, \\
\gamma_{2}(m)=2 \gamma_{2}(m-1)-3 \gamma_{2}(m-4)+2 \gamma_{2}(m-5)+ \begin{cases}0 & \text { if } m \text { is even } \\
1 & \text { otherwise }\end{cases} \\
H(m)=2\left[\frac{m-2}{2}\right] .
\end{gathered}
$$

For CA- $156_{1-0}(m)$

$$
\begin{gathered}
\gamma_{1}(m)= \begin{cases}0 & \text { if } m \text { is odd } \\
1 & \text { otherwise }\end{cases} \\
\gamma_{2}(m)=\gamma_{2}(m-2)+2 \gamma_{2}(m-3)+\gamma_{1}(m-3), \\
H(m)_{1-0}=2\left[\frac{m}{2}\right] .
\end{gathered}
$$




\section{Dynamical Behaviors}

In this section, we study behaviors of $C A-156_{\alpha-\beta}(m)$ in terms of limit cycles, their numbers and transient lengths.

\subsection{Boundary Condition 1-0}

In this subsection we investigate behaviors of CA-156 $6_{1-0}(\mathrm{~m})$, and we prove the formulas stated in section 2 .

Lemma 1 Let $x$ be a configuration and $y=\delta(x)$. Then if $x_{i} x_{i+1}=01$, then $y_{i} y_{i+1}=$ 01 where $1 \leq i \leq m$.

Proof. It is trivial.

Lemma 2 The configuration $x$ is a fixed point if and only if $1 x 0$ contains no subsequences 11 and 00 .

Proof. (necessary condition) First, suppose that $1 x 0$ contains the subsequence 11. As the boundary condition is $1-0,1 x 0$ contains the subsequence 110 . So $x$ is not a fixed point as $f(110)=0$. Next, suppose that $1 x 0$ contains the subsequence 00 . As the boundary condition is $1-0,1 x 0$ contains the subsequence 100 . So $x$ is not a fixed point as $f(100)=1$.

(sufficient condition) Let $y=\delta(x)$. Suppose that $x$ is not a fixed point, then there exists an integer $i$ with $1 \leq i \leq m$ such that $x_{i} \neq y_{i}$. If $x_{i}=0$ and $y_{i}=1$, it follows that only $x_{i-1} x_{i} x_{i+1}=100$ for $y_{i}=1$. So $1 x 0$ contains the subsequence 00 . If $x_{i}=1$ and $y_{i}=0$, it follows that only $x_{i-1} x_{i} x_{i+1}=110$ for $y_{i}=0$. So $1 x 0$ contains the subsequence 11 .

Lemma 3 Let $x$ be a configuration. Then $x=\delta^{2}(x)$ if and only if $1 x 0$ contains no subsequences 111, 000 and 1100 .

Proof. Let $y=\delta(x)$ and $z=\delta^{2}(x)$.

(necessary condition) First, suppose that $x$ contains the subsequence 111 . The boundary condition is $1-0$, so there exists an integer $i$ with $1 \leq i \leq m-1$ such that $x_{i-1} x_{i} x_{i+1} x_{i+2}=1110$. As $f(* 11)=1$ and $f(110)=0$, we have $y_{i-1} y_{i} y_{i+1}=110$ and $z_{i+1}=0$. Hence $x \neq \delta^{2}(x)$. Next, suppose that $x$ contains the subsequence 000 . The boundary condition is $1-0$, so there exists an integer $i$ with $1 \leq i \leq m-1$ such that $x_{i-1} x_{i} x_{i+1} x_{i+2}=1000$. As $f(100)=1$ and $f(00 *)=0$, we have $y_{i} y_{i+1} y_{i+2}=100$ and $z_{i+1}=1$. Hence $x \neq \delta^{2}(x)$.

(sufficient condition) Suppose that $x \neq \delta^{2}(x)$. Then there exists an integer $i$ such that $x_{i} \neq z_{i}$ where $1 \leq i \leq m$. First we let $x_{i}=1$ and $z_{i}=0$. Then for $z_{i}=0$, it follows that $y_{i-1} y_{i} y_{i+1}=110,101,001$ or 000 . 
1. If $y_{i-1} y_{i} y_{i+1}=110$, then for $y_{i}=1$, it follows that $x_{i-1} x_{i} x_{i+1}=111,011$ and 010. But $x_{i-1} x_{i} x_{i+1}=011$ and 010 contradict $y_{i-1} y_{i} y_{i+1}=110$. So we have $x_{i-1} x_{i} x_{i+1}=111$.

2. If $y_{i-1} y_{i} y_{i+1}=101$, then for $y_{i}=0$, it follows that $x_{i-1} x_{i} x_{i+1}=110$. Then for $y_{i+1}=1$, we have $x_{i+2}=0$. So $x$ contains 1100. $x_{i+2}=0$.

3. If $y_{i-1} y_{i} y_{i+1}=001$, then for $y_{i}=0$, it follows that $x_{i-1} x_{i} x_{i+1}=110$. But $x_{i-1} x_{i} x_{i+1}=110$ contradict $y_{i-1} y_{i} y_{i+1}=001$.

4. If $y_{i-1} y_{i} y_{i+1}=000$, then for $y_{i}=0$, it follows that $x_{i-1} x_{i} x_{i+1}=110$. But $x_{i-1} x_{i} x_{i+1}=110$ contradict $y_{i-1} y_{i} y_{i+1}=000$.

Next we let $x_{i}=0$ and $z_{i}=1$. Then for $z_{i}=1$, it follows that $y_{i-1} y_{i} y_{i+1}=$ $111,100,011$ or 010 .

1. If $y_{i-1} y_{i} y_{i+1}=111$, then for $y_{i}=1$, it follows that only $x_{i-1} x_{i} x_{i+1}=100$. But as $f(00 *)=0, x_{i-1} x_{i} x_{i+1}=100$ contradicts $y_{i+1}=1$.

2. If $y_{i-1} y_{i} y_{i+1}=100$, then for $y_{i-1} y_{i}=10$, it follows that $x_{i-2} x_{i-1} x_{i} x_{i+1}=1000$ or 1001. But $x_{i-2} x_{i-1} x_{i} x_{i+1}=1001$ contradicts $y_{i+1}=0$ as $f(01 *)=1$. So $x_{i-2} x_{i-1} x_{i} x_{i+1}=1000$.

3. If $y_{i-1} y_{i} y_{i+1}=01 *$, then for $y_{i-1} y_{i}=01$, it follows that only $x_{i-2} x_{i-1} x_{i} x_{i+1}=$ 1100

Therefore if $x \neq \delta^{2}(x)$, then $1 x 0$ contains the subsequences 111,000 and 1100 .

From lemma 2 and lemma 3 we have that the configuration $x$ is on a limit cycle of period length 2 if and only if $1 x 0$ contains the subsequences 11 or 00 , and does not contain the subsequences 111,000 and 1100 .

Lemma 4 Let $x$ be a configuration and $y=\delta(x)$. Then if $y_{i-1}=y_{i}=y_{i+1}$, then $x_{i-1}=x_{i}=x_{i+1}$.

Proof. First, we assume $y_{i-1}=y_{i}=y_{i+1}=1$. For $y_{i}=1$, it follows that $x_{i-1} x_{i} x_{i+1}=111,100,011$ and $010 . x_{i-1} x_{i} x_{i+1}=100$ contradicts $y_{i+1}=1$ as $f(00 *)=$ $0 . x_{i-1} x_{i} x_{i+1}=011$ and 010 contradict $y_{i-1}=1$ as $f(* 01)=0$. If $x_{i-1} x_{i} x_{i+1}=111$, then we have $y_{i-1} y_{i} y_{i+1}=111$ from assumption that $x_{i+2}=1$. So if $y_{i-1}=y_{i}=$ $y_{i+1}=1$, then $x_{i-1}=x_{i}=x_{i+1}=1$.

Next, we assume $y_{i-1}=y_{i}=y_{i+1}=0$. For $y_{i}=0$, it follows that $x_{i-1} x_{i} x_{i+1}=$ 110,101,001 and 000. $x_{i-1} x_{i} x_{i+1}=110$ contradicts $y_{i-1}=0$ as $f(* 11)=1 . x_{i-1} x_{i} x_{i+1}=$ 101 and 001 contradicts $y_{i+1}=0$ as $f(01 *)=1$. If $x_{i-1} x_{i} x_{i+1}=000$, then we have $y_{i-1} y_{i} y_{i+1}=000$ from assumption that $x_{i-2=0}$. So if $y_{i-1}=y_{i}=y_{i+1}=0$, then $x_{i-1}=x_{i}=x_{i+1}=0$.

Lemma 5 Let $x$ be a configuration and $y=\delta(x)$. Then if $\delta(x)$ contains the subsequence 1100 , then $x$ contains the subsequence 111 or 000 . 
Proof. Let $y=\delta(x)$. Suppose that $y$ contain the subsequence 1100, and let $y_{i-1} y_{i} y_{i+1} y_{i+2}=1100$. For $y_{i-1} y_{i}=11$, it follows that $x_{i-2} x_{i-1} x_{i} x_{i+1}=1111$ and 0100 . If $x_{i-2} x_{i-1} x_{i} x_{i+1}=1111$, then for $y_{i+1} y i+2=00$, it follows that $x_{i+2} x_{i+3}=01$. If $x_{i-2} x_{i-1} x_{i} x_{i+1}=0100$, then for $y_{i+1} y i+2=00$, it follows that $x_{i+2} x_{i+3}=00$. Hence if $\delta(x)$ contains 1100 , then $x$ contains 111 or 000 .

Lemma 6 The following hold;

- If $x_{i} x_{i+1} \cdots x_{i+s}=10^{s}$, then $y_{i+1} y_{i+2} \cdots y_{i+s}=10^{s-1}$ where $s \leq 2$.

- If $x_{i} x_{i+1} \cdots x_{i+s}=1^{s} 0$, then $y_{i+1} y_{i+2} \cdots y_{i+s}=1^{s-1} 0$ where $s \leq 2$.

Proof. It is trivial.

Proposition 1 All 2-cycle of $C A-156_{1-0}(m)$ can be constructed without redundancy as follows;

1. If $x_{1} x_{2} \cdots x_{m-2}$ and $y_{1} y_{2} \cdots y_{m-2}$ form a D-cycle, then $x_{1} x_{2} \cdots x_{m-2} 01$ and $y_{1} y_{2} \cdots y_{m-2} 01$ form a 2 -cycle.

D. If $x_{1} x_{2} \cdots x_{m-3}$ and $y_{1} y_{2} \cdots y_{m-3}$ form a D-cycle, then $x_{1} x_{2} \cdots x_{m-2} 010$ and $y_{1} y_{2} \cdots y_{m-2} 011$ form a D-cycle.

3. If $x_{1} x_{2} \cdots x_{m-3}$ is a fixed point, then $x_{1} x_{2} \cdots x_{m-3} 010$ and $x_{1} x_{2} \cdots x_{m-3} 011$ form a D-cycle.

Proof. 1,2,3: From lemma 2 and lemma 3, it is clear. 4: First, configurations which are constructed form a limit cycle of period length 2 and don't overlap each other clearly.

Next, let $a$ and $b$ be configurations which form a limit cycle of period length 2 . We look at last 2 cells. In case $a_{m-1} a_{m}=00$, then $a$ doesn't form a limit cycle of period length 2. In case $a_{m-1} a_{m}=01$, then we have $b_{m-1} b_{m}=01$ as $a$ and $b$ form a limit cycle. So $1 a_{1} a_{2} \cdots a_{m-2} 0$ and $1 b_{1} b_{2} \cdots b_{m-2} 0$ contain the subsequences 11 or 00 and don't contain 111,000 and 1100. So $a_{1} a_{2} \cdots a_{m-2}$ and $b_{1} b_{2} \cdots b_{m-2}$ form a limit cycle of period length 2 of $C A-156(m-2)_{1-0}$. In case $a_{m-1} a_{m}=10$, then if $a_{m-2}=1 \mathrm{a}$ doesn't form a 2 -cycle. If $a_{m-2}=0$, then we have $b_{m-2} b_{m-1} b_{m}=011$. So $1 a_{1} a_{2} \cdots a_{m-3} 0$ and $1 b_{1} b_{2} \cdots b_{m-3} 0$ don't contain 111,000 and 1100 . Hence $a_{1} a_{2} \cdots a_{m-3}$ and $b_{1} b_{2} \cdots b_{m-3}$ form a limit cycle of period length 1 or 2 of $C A-156(m-3)_{1-0}$. In case $a_{m-1} a_{m}=10$, then if $a_{m-2}=1, a$ doesn't form a limit cycle of period length 2 . If $a_{m-2}=0$, then we have $b_{m-2} b_{m-1} b_{m}=011$. So $1 a_{1} a_{2} \cdots a_{m-3} 0$ and $1 b_{1} b_{2} \cdots b_{m-3} 0$ don't contain 111,000 and 1100. Hence $a_{1} a_{2} \cdots a_{m-3}$ and $b_{1} b_{2} \cdots b_{m-3}$ form a limit cycle of period length 1 or 2 of $C A-156(m-3)_{1-0}$.

So all configurations which form a limit cycle of period length 2 are constructed without overlap.

By the above discussion we get the following theorem. 
Theorem $1 C A-156_{1-0}(m)$ has limit cycles of period length 1 and 2 , and for the number of limit cycles the following hold;

$$
\begin{gathered}
\gamma_{1}(m)= \begin{cases}0 & \text { if } m \text { is odd } \\
1 & \text { otherwise }\end{cases} \\
\gamma_{2}(m)=\gamma_{2}(m-2)+2 \gamma_{2}(m-3)+\gamma_{1}(m-3) .
\end{gathered}
$$

And for the transient length of $C A-156_{1-0}(m)$ the following holds;

$$
H(m)_{1-0}=2\left[\frac{m}{2}\right] .
$$

Proof. First, as for the formulae of numbers of limit cycles, it is clear by proposition 1.

Next, as for the formula of transient length, let $x(k)=\delta^{k}(x)$. First case, when the configuration $1 x 0$ contain $0^{s}$, let $x_{i+1} x_{i+2} \cdots x_{i+s}=0^{s}$ and $x_{i+s+1}=1$ or $i+s=m+1$. In case $x_{i-1} x_{i}=01$ or $i=0$.

1. If $s$ is even, then we have $x_{i+1}(s-3) x_{i+2}(s-3) \cdots x_{i+s}(s-3)=0101 \cdots 011000$. So $x_{i}(s-2) x_{i+2}(s-2) \cdots x_{i+s+1}(s-2)$ doesn't contain 111,000 and 1100 .

2. If $s$ is even, then we have $x_{i+1}(s-2) x_{i+2}(s-2) \cdots x_{i+s}(s-2)=0101 \cdots 01100$. So $x_{i}(s-1) x_{i+2}(s-1) \cdots x_{i+s+1}(s-1)$ doesn't contain 111,000 and 1100 .

In case $x_{i-1} x_{i}=11$.

1. If $s$ is even, then we have $x_{i+1}(s-2) x_{i+2}(s-2) \cdots x_{i+s}(s-1)=0101 \cdots 011000$. So $x_{i}(s-1) x_{i+2}(s-1) \cdots x_{i+s+1}(s-1)$ doesn't contain 111,000 and 1100 .

2. If $s$ is even, then we have $x_{i+1}(s-3) x_{i+2}(s-3) \cdots x_{i+s}(s-3)=0101 \cdots 01100$. So $x_{i}(s-2) x_{i+2}(s-2) \cdots x_{i+s+1}(s-2)$ doesn't contain 111,000 and 1100 .

Second case, when the configuration $1 x 0$ contain $1^{s}$, let $x_{i+1} x_{i+2} \cdots x_{i+s}=1^{s}$ and $x_{i}=0$ or $i+1=0$.

In case $x_{i-1} x_{i}=01$ or $i+s+1=m+1$.

1. If $s$ is even, then we have $x_{i+1}(s-3) x_{i+2}(s-3) \cdots x_{i+s}(s-3)=11100101 \cdots 01$. So $x_{i}(s-2) x_{i+2}(s-2) \cdots x_{i+s+1}(s-2)$ doesn't contain 111,000 and 1100 .

2. If $s$ is odd, then we have $x_{i+1}(s-2) x_{i+2}(s-2) \cdots x_{i+s}(s-2)=1100101 \cdots 01$. So $x_{i}(s-1) x_{i+2}(s-1) \cdots x_{i+s+1}(s-1)$ doesn't contain 111,000 and 1100 .

In case $x_{i-1} x_{i}=00$.

1. If $s$ is even, then we have $x_{i+1}(s-2) x_{i+2}(s-2) \cdots x_{i+s}(s-2)=1100101 \cdots 010$. So $x_{i}(s-1) x_{i+2}(s-1) \cdots x_{i+s+1}(s-1)$ doesn't contain 111,000 and 1100 .

2. If $s$ is odd, then we have $x_{i+1}(s-3) x_{i+2}(s-3) \cdots x_{i+s}(s-3)=11100101 \cdots 010$. So $x_{i}(s-2) x_{i+2}(s-2) \cdots x_{i+s+1}(s-2)$ doesn't contain 111,000 and 1100 .

So when $1 x 0$ contain $1^{m+1}$ or $0^{m+1}, h(x)$ is maximal. So if $m$ is even, then $H(m)_{1-0}=$ $m$ and if $m$ is odd, then $H(m)_{1-0}=m-1$. Hence $H(m)=2\left[\frac{m}{2}\right]$. 


\subsection{Boundary Condition 0-0 and 1-1}

The reverse rule of the symmetric rule of rule 156 is rule 156 . So CA-156 $6_{0-0}(m)$ and CA-156 $6_{1-1}(\mathrm{~m})$ are isomorphic. In this subsection we investigate behaviors of CA- $156_{1-=1}(m)$ only.

Lemma 7 Let $x$ be a configuration and $y=\delta(x)$. If $x_{m-i} x_{m-i+1} \cdots x_{m}=01^{i}$, then $y_{m-i} y_{m-i+1} \cdots y_{m}=01^{i}$ where $0 \leq i \leq m-1$.

Proof. It is trivial.

From lemma 7 , behaviors of CA-156 $6_{1-1}(m)$ is isomorphic to behaviors of CA-156 $6_{1-0}(m-$ $i-1)$. So we get the following theorem from theorem 1

Theorem 2 CA-156 $1-1(m)$ has limit cycles of period length 1 and 2, and for the number of limit cycles the following hold;

$$
\begin{gathered}
\gamma_{1}(m)=\left[\frac{m-1}{2}\right]+2, \\
\gamma_{2}(m)=\gamma_{2}(m-1)+\gamma_{2}(m-2)+\gamma_{2}(m-3)-2 \gamma_{2}(m-4)+\left\{\begin{array}{ll}
0 & \text { if } m \text { is even } \\
1 & \text { otherwise }
\end{array} .\right.
\end{gathered}
$$

And for the transient length of $C A-156_{1-1}(m)$ the following holds;

$$
H(m)=2\left[\frac{m-1}{2}\right] .
$$

Proof. If $x=1^{m}$ or $01^{m-1}$, then $\delta(x)=x$. If $x \neq 1^{m}$ and $01^{m-1}$, then there exists a positive integer $j$ such that $j=\max _{x_{i}=0}\{i\}$. So for any configuration $x$ such that $x \neq 1^{m}$ and $01^{m-1} x_{m-j} x_{m-j+1} \cdots x_{m}=01^{j}$ where $0 \leq j \leq m-2$. By lemma 7 , behaviors of $x_{1} x_{2} \cdots x_{m-i-1}$ are same as it of CA-156 $15-0(m-i-1)$. So we have

$$
\gamma_{1}(m)_{1-1}=\gamma_{1}(m-1)_{1-0}+\cdots+\gamma_{1}(1)_{1-0}+2
$$

and

$$
\gamma_{2}(m)_{1-1}=\gamma_{2}(m-1)_{1-0}+\cdots+\gamma_{2}(1)_{1-0}
$$

By theorem 1, we have

$$
\gamma_{1}(m)_{1-1}=\left[\frac{m-1}{2}\right]+2
$$

and

$$
\gamma_{2}(m)_{1-1}=\gamma_{2}(m-1)_{1-1}+\gamma_{2}(m-2)_{1-1}+\gamma_{2}(m-3)_{1-1}-2 \gamma_{2}(m-4)_{1-1}+0 / 1 .
$$

As $\max \left\{h(x) \mid x_{m-i} x_{m-i+1} \cdots x_{m}=01^{i}\right\}$ equals to transient length of CA-156 $6_{1-0}(m-$ $i-1)$. So $H(m)=2\left[\frac{m-1}{2}\right]$. 


\subsection{Boundary Condition 0-1}

In this subsection we investigate behaviors of CA-156 $6_{0-1}(m)$.

Lemma 8 Let $x$ be a configuration and $y=\delta(x)$. If $x_{1} x_{2} \cdots x_{i+1}=0^{i} 1$, then $y_{1} y_{2} \cdots y_{i+1}=0^{i} 1$ where $0 \leq i \leq m-1$.

Proof. It is trivial.

From the above lemma CA-156 $6_{0-1}(m)$ and CA-156 $6_{1-1}(m-i-2)$ are isomorphic each other. So we get the following theorem.

Theorem $3 C A-156_{0-1}(m)$ has limit cycles of period length 1 and 2 , and for the number of limit cycles the following hold;

$$
\begin{gathered}
\gamma_{1}(m)=\gamma_{1}(m-1)+\gamma_{1}(m-2)-\gamma_{1}(m-3)+1 \\
\gamma_{2}(m)=2 \gamma_{2}(m-1)-3 \gamma_{2}(m-4)+2 \gamma_{2}(m-5)+\left\{\begin{array}{ll}
0 & \text { if } m \text { is even } \\
1 & \text { otherwise }
\end{array} .\right.
\end{gathered}
$$

And for the transient length of $C A-156_{0-1}(m)$ the following holds;

$$
H(m)=2\left[\frac{m-2}{2}\right] .
$$

\section{Conclusion}

In this paper we investigated behaviors of $C A-156_{\alpha-\beta}(m)$ in terms of limit cycles and their numbers, and transient length, and proved the formulas of the number of limit cycles and transient lengths.

\section{References}

[1] S. Wolfram: Theory and Applications of Cellular Automata, World Scientific, Singapore, 1986

[2] Y.Kawahara,S.Kumamoto,Y.Mizoguchi,M.Nohmi,H.Ohtuka and T.Shoudai: Period lengths of cellular automata on square lattices with rule 90, J.Math.Phys.36(3),March 1995

[3] H.Lee and Y.Kawahara: On dynamical behaviors of cellular automata CA-60, Bull.Informatics and Cybernetics 25,22-27,1992

[4] R. Shingai.: The Maximum Period Realized in 1-D Uniform Neural Networks, Trans.IECE,Japan, E61,1978,804-808

[5] M. Kawanaka, M. Tsunoyama and S. Naito: A Fault-Tolerant Parallel Processor Model by a Two Dimensional Linear Cellular Automaton 
[6] H.Lee and Y.Kawahara: Transition Diagrams of Finite Cellular Automata, Bull. Inform. Cybernet. 28(1996)47-69

[7] T. Sato: On Behaviors of Cellular Automata With Rule 27, Kyushu J. Math., Vol.50, 1996, pp.133-152

[8] S. Inokuchi, T. Sato, A. Hara, S. Kumamoto, H. Lee, Y. Kawahara: Computational Analysis of Cellular Automata with Triplet Transition Rule, Research Report on ISEE of Kyushu Univ., Vol.1, No.1, 1996 